\title{
Maternal Health, Neonatology, and Perinatology reviewer acknowledgement 2015
}

Tonse Nk Raju

\section{Contributing reviewers}

The editor of Maternal Health, Neonatology, and Perinatology would like to thank all of the reviewers who have contributed to the journal in Volume 1 (2015).

\section{Alexandria Hill}

United States of America

Alireza Shamshiraz

United States of America

Anup Katheria

United States of America

Ashley Williams

United States of America

Carlos Blanco

Ireland

Christina Herrera

United States of America

David Hutchon

United Kingdom

Devaraj Sambalingam

United States of America

Don McCurnin

United States of America

Elizabeth McClure

United States of America

Heike Rabe

United Kingdom
Jayashree Ramasethu

United States of America

Jonathan Samet

United States of America

Jorge Tolosa

United States of America

Kelli Barbour

United States of America

Malissa Parisi

United States of America

Martin Keszler

United States of America

Matthew Laughon

United States of America

Nithi Fernandes

United States of America

Payam Vali

United States of America

Praveen Chandrasekharan

United States of America

Robert Silver

United States of America
Rodriguez Christina

United States of America

Roland Hentschel

Germany

Rosemary Higgins

United States of America

Ruth Grunau

Canada

Satyan Lakshminrusimha

United States of America

\section{Stuart Hooper}

Australia

Vikramaditya Dumpa

United States of America

Vinay Sharma

United States of America

Ware Branch

United States of America

William Zorn

United States of America

Yawei Zhang

United States of America

Correspondence: mhnpjournal@biomedcentral.com

National Institutes of Health, 31 Center Drive, Bethesda, MD 20892-2425, USA 